\title{
Comparative Study of Heart Rate Variability in Patients with Schizophrenia, Bipolar Disorder, Post-traumatic Stress Disorder, or Major Depressive Disorder
}

\author{
Eunok Moon ${ }^{1}$, Seung-Hwan Lee ${ }^{1,2}$, Do-Hyung Kim², Boram Hwang ${ }^{2}$ \\ ${ }^{1}$ Clinical Emotion and Cognition Research Laboratory, ${ }^{2}$ Department of Psychiatry, Inje University IIsan Paik Hospital, Goyang, Korea
}

\begin{abstract}
Objective: Heart rate variability (HRV) changes as a function of psychiatric illness. This study aimed to evaluate HRV among patients with various psychiatric disorders.

Methods: The present study recruited patients with schizophrenia $(n=35)$, bipolar disorder $(n=41)$, post-traumatic stress disorder (PTSD; $n=34)$, or major depressive disorder $(n=34)$ as well as healthy controls ( $n=27)$. The time-domain analysis (the standard deviation of all RR intervals [SDNN] and the square root of the mean squared differences of successive normal sinus intervals [RMSSD]), the frequency-domain analysis (very low frequency, low frequency [LF], high frequency [HF], and total power [TP]), and a non-linear complexity measure the approximate entropy were computed.

Results: SDNN and HF were significantly reduced in patients with schizophrenia compared with healthy controls. SDNN, RMSSD, TP, LF, and HF were significantly reduced in bipolar patients compared with healthy controls. HF was significantly reduced in PTSD patients compared with healthy controls.

Conclusion: Our findings indicate that HRV is not sufficiently powerful to discriminate among various psychiatric illnesses. However, our results suggest that HRV, particularly HF, could be used as a tool for discriminating between psychiatric patients and healthy controls.
\end{abstract}

KEY WORDS: Heart rate variability; Schizophrenia; Bipolar disorder; Post traumatic stress disorder; Major depressive disorder.

\section{INTRODUCTION}

Autonomic nervous system (ANS) functioning depends on a balance between the activities of the sympathetic and parasympathetic nervous systems in the human body. Under resting conditions, parasympathetic activity is dominant, whereas sympathetic activity is dominant under anxious and physically active conditions. To maintain this balance, the parasympathetic system needs to be very sensitive and respond quickly to external and internal environmental changes. This balance can be measured using heart rate variability (HRV).

$\mathrm{HRV}$ is analyzed using time-domain analysis, frequency-domain analysis, and a non-linear complexity measure. In the time-domain analysis, the standard deviation of all RR intervals (SDNN) and the square root of the mean squared differences of successive normal sinus in-

\footnotetext{
Received: April 4, 2013 / Revised: May 27, 2013

Accepted: June 10, 2013

Address for correspondence: Seung-Hwan Lee, PhD

Department of Psychiatry, Inje University IIsan Paik Hospital, 170

Juhwa-ro, IIsanseo-gu, Goyang 411-706, Korea

Tel: +82-31-910-7260, Fax: +82-31-910-7268

E-mail: Ishpss@paik.ac.kr
}

tervals (RMSSD) are computed according to standardized procedures. ${ }^{1,2)}$ SDNN is therefore a measure of heart rate change and reflects total variability during the recording period. RMSSD is sensitive to high-frequency heart period fluctuations in the respiratory frequency range and is used as an index of vagal cardiac control. ${ }^{3)}$ In the frequency-domain analysis, the power spectra of three frequency bands are calculated: very low frequency (VLF; 0.005-0.040 Hz), low frequency (LF; 0.04-0.15 Hz), high frequency (HF; $0.15-0.40 \mathrm{~Hz}$ ), and total power (TP), including VLF, LF, and HF.1) For the non-linear complexity measure, the approximate entropy (ApEn) is calculated. ${ }^{4)}$ The ApEn is a parameter that was developed to quantify the degree of regularity versus unpredictability in a higher dimensional attractor reconstructed from a time series, such as the instantaneous heart rate time series. ${ }^{5,6)}$ A lower ApEn value reflects a higher degree of regularity, and the higher the entropy value, the more unpredictable the time series. $^{5,6)}$

Sympathetic activity acts at a lower frequency (LF: $0.04-0.15 \mathrm{~Hz})^{7)}$ of heart rate, and parasympathetic activity produces changes in heart rate at a relatively higher fre-

(c) This is an Open-Access article distributed under the terms of the Creative Commons Attribution Non-Commercial License (http://creativecommons.org/licenses/by-nc/3.0) which permits unrestricted non-commercial use, distribution, and reproduction in any medium, provided the original work is properly cited. 
quency (HF: 0.15-0.4 Hz) than does sympathetic activity. ANS activity can be mapped by variations in the high-frequency bandwidth of the heart rate (i.e., parasympathetic activity), and $\mathrm{HRV}$ is therefore often used as a convenient and non-invasive index of ANS activity. High-frequency HRV (HF-HRV) is widely accepted as representing the flexibility of vagal (parasympathetic) tone and the general capacity of the ANS to respond to changing environmental conditions in an adaptive way. ${ }^{8)}$ Thus, HRV may reflect the pathophysiology of various psychiatric disorders.

Although many researchers have conducted HRV studies with patients with major depressive disorder (MDD), the results of these studies are in conflict. ${ }^{9-12)}$ Most found reduced vagal modulation in patients with $\mathrm{MDD},{ }^{13-16)}$ whereas others did not find differences in HRV between patients and controls. ${ }^{11,17,18)}$ Antidepressant medication is assumed to have mixed effects on HRV. Research papers on this issue illustrate that changes in autonomic functioning depend on the type of antidepressant. Tricyclic antidepressants and serotonin-norepinephrine reuptake inhibitors tend to reduce HRV ${ }^{14,19,20)}$ whereas selective serotonin reuptake inhibitors (SSRIs) increase HRV. ${ }^{19,21)}$

Lower HF-HRV has been reported in conditions characterized by deficient emotional regulation, such as high trait anxiety ${ }^{22)}$ and negative affect. ${ }^{23)}$ This diminished HF-HRV is observed across anxiety disorders (e.g., panic, generalized anxiety, social anxiety, and obsessive-compulsive disorder) relative to healthy controls. ${ }^{24)}$ Further, patients with post-traumatic stress disorder (PTSD) have reduced HRV modulation. ${ }^{25-28)}$

An increasing number of reports have also observed HRV dysfunction in schizophrenia. ${ }^{29-31)}$ Two studies reported that drug-naïve patients with schizophrenia exhibit decreased HF-HRV power compared with healthy subjects but no differences in LF power were observed. ${ }^{29,31)}$ These data suggest that schizophrenia is characterized by a decrease in parasympathetic activity independent of medication effects. Decreased vagal tone in patients with schizophrenia is significantly correlated with symptom severity. ${ }^{32,33)}$

One report examined HRV in a population of subjects with euthymic bipolar disorder and reported a decrease in SDNN, a decrease in the LF/HF ratio, and an increase in HF power compared with healthy subjects, ${ }^{34)}$ indicating an increase in vagal tone. Henry et al $^{35)}$ reported that patients in the manic phase of bipolar disorder exhibit a significant decrease in HRV compared with age- and sex-matched healthy subjects. An increase in the ratio of sympathetic to parasympathetic activity is associated with more severe manic symptoms and unusual thought content.

Although studies comparing HRV in patients with specific psychiatric disorders with control subjects have been conducted, we know of no previous studies evaluating and comparing the HRV of patients with various psychiatric disorders (i.e., MDD, anxiety disorder, bipolar disorder, schizophrenia) with that of healthy controls. Comparing the HRV pattern associated with various psychiatric illnesses under the same examination conditions could provide clinicians with useful information about disease characteristics. The aim of our study was to assess the pattern of resting-state HRV response demonstrated by patients with various psychiatric disorders. We aimed to determine if HRV could be used as a way to discriminate among various psychiatric disorders.

\section{METHODS}

\section{Subjects}

A total of 171 subjects between the ages of 18 and 60 years participated in this study. The sample included patients with schizophrenia $(\mathrm{n}=35)$, bipolar disorder $(\mathrm{n}=41)$, $\operatorname{PTSD}(\mathrm{n}=34)$, and MDD $(\mathrm{n}=34)$ as well as healthy controls $(n=27)$. All of the patients met criteria for Axis I diagnoses in the Diagnostic and Statistical Manual of Mental Disorders, 4th edition (DSM-IV; American Psychiatric Association, 1994). Healthy control subjects were recruited from the local community via newspaper advertisements. After initial screening to rule out Axis I disorders, potential healthy controls were interviewed using the Structured Clinical Interview for DSM-IV Axis II Disorders $^{36)}$ and were excluded if they had any kind of personality disorder.

All patients with MDD and PTSD were being treated with SSRIs (i.e., fluoxetine, escitalopram, or paroxetine). Patients with schizophrenia were being treated with atypical antipsychotic medication (i.e., olanzapine, risperidone, quetiapine, or aripiprazole). Patients with bipolar disorder were being treated with mood stabilizing agents (i.e., lithium, valproate, or lamotrigine) with or without atypical antipsychotics. None of the subjects had a history of neurological disorder, substance abuse, mental retardation, or brain trauma. Written informed consent was obtained from all subjects, and the study protocol was approved by the Ethics Committee of Inje University Ilsan Paik Hospital. 


\section{Hear Rate Variability}

HRV measurements were taken 0 to 7 days after starting medication. Smoking and coffee consumption were prohibited for at least $6 \mathrm{~h}$ before measurement. After each subject was allowed to adapt to the experimental conditions for approximately $10 \mathrm{~min}, 5$-min single-channel (3-lead) electrocardiogram (ECG) recordings were performed in the seated position at complete rest using the HRV analyzer (SA-3000P) from Medicore Co., Ltd (Seoul, Korea). The ECG signal was amplified and digitized, and the RR interval time series was generated using the automatic scheme to detect the R peak in the ECG using previously published methods. ${ }^{1)}$

\section{Statistical Analysis}

A chi-square test for group differences according to sex and one-way analyses of variance (ANOVAs) were used to examine differences in demographic variables using SPSS 18.0 for Windows. A multivariate ANOVA was used with age and sex as covariates to analyze HRV items.
As a post hoc analysis, between- group estimated marginal means were compared with confidence interval adjustment using the Bonferroni method. Spearman's correlation analysis was used to analyze relationships between HRV items and symptom severity for each psychiatric disorder.

\section{RESULTS}

\section{Demographic Variables}

Demographic characteristics are presented in Table 1. The sex and age distributions of the groups significantly differed as subjects were recruited from a hospital setting.

\section{HRV Variables}

All HRV variables are presented in Table 2.

\section{SDNN}

We found a significant main effect of SDNN ( $F[4$, $165]=3.55, p=0.008)$. Post hoc analysis revealed two sig-

Table 1. Demographic characteristics and symptoms ratings

\begin{tabular}{|c|c|c|c|c|c|c|}
\hline & Schizophrenia $(n=35)$ & Bipolar disorder $(n=41)$ & PTSD $(n=34)$ & $\operatorname{MDD}(n=34)$ & Healthycontrol $(n=27)$ & $F$ value \\
\hline Sex (male/female) & $17 / 18$ & $11 / 30$ & $19 / 15$ & $6 / 28$ & $6 / 21$ & $0.002^{*}$ \\
\hline Age $(y r)$ & $33.89(12.03)$ & $36.20(12.43)$ & $40.82(14.64)$ & $57.82(15.86)^{\dagger}$ & $34.30(11.09)$ & $18.93^{\text {}}$ \\
\hline Illness duration (yr) & $6.02(6.24)$ & $5.28(5.87)$ & $0.87(1.14)$ & $2.98(3.45)$ & & $8.596^{\ddagger}$ \\
\hline \multicolumn{7}{|l|}{ PANSS score } \\
\hline Positive & $25.14(8.66)$ & $25.81(7.60)$ & & & & \\
\hline Negative & $21.06(8.24)$ & $14.76(4.94)$ & & & & \\
\hline General & $49.86(10.76)$ & $48.71(6.97)$ & & & & \\
\hline Total & $96.06(19.17)$ & $89.30(15.22)$ & & & & \\
\hline YMRS score & & $19.15(8.58)$ & & & & \\
\hline HAMA score & & & $24.09(7.35)$ & & & \\
\hline HAMD score & & & & $25.76(7.04)$ & & \\
\hline
\end{tabular}

Values are presented as number only or mean (standard deviation).

${ }^{*}$ Chi Square $p$ value; ${ }^{\dagger} p<0.05,{ }^{\dagger} p<0.001$.

PTSD, post-traumatic stress disorder; MDD, major depressive disorder; PANSS, Positive and Negative Syndrome Scale; YMRS, Young Mania Rating Scale; HAMA, Hamilton Anxiety Rating Scale; HAMD, Hamilton Depression Rating Scale.

Table 2. Estimated mean values and standard error of HRV items

\begin{tabular}{|c|c|c|c|c|c|c|}
\hline HRV Items & Schizophrenia $(n=35)$ & Bipolar disorder $(n=41)$ & PTSD $(n=34)$ & $\operatorname{MDD}(n=34)$ & Healthy control $(n=27)$ & $F$ value \\
\hline SDNN & $30.05(1.95)$ & $28.80(1.78)$ & $31.21(1.96)$ & $33.71(2.23)$ & $38.57(2.22)$ & $3.55^{\star}$ \\
\hline RMSSD & $23.99(2.08)$ & $20.30(1.89)$ & $22.36(2.09)$ & $25.99(2.37)$ & $30.27(2.35)$ & $3.17^{\dagger}$ \\
\hline VLF & $335.96(62.76)$ & $363.65(57.27)$ & $404.21(63.10)$ & $421.23(71.54)$ & $501.26(71.13)$ & 0.92 \\
\hline LF & $228.63(39.43)$ & $163.04(35.99)$ & $178.86(39.65)$ & $239.35(44.95)$ & $335.48(44.70)$ & $2.72^{\dagger}$ \\
\hline $\mathrm{HF}$ & $197.24(31.82)$ & $121.35(29.04)$ & $157.56(31.99)$ & $159.16(36.27)$ & $332.83(36.07)$ & $5.86^{\ddagger}$ \\
\hline $\mathrm{TP}$ & $761.08(104.47)$ & $651.42(95.33)$ & $735.71(105.03)$ & $843.67(119.02)$ & $1174.69(118.41)$ & $3.38^{\dagger}$ \\
\hline $\mathrm{LF} / \mathrm{HF}$ & $1.55(0.31)$ & $2.00(0.28)$ & $2.47(0.31)$ & $1.88(0.35)$ & $1.56(0.35)$ & 1.423 \\
\hline ApEn & $1.19(0.02)$ & $1.17(0.02)$ & $1.15(0.02)$ & $1.11(0.02)$ & $1.13(0.02)$ & 1.64 \\
\hline
\end{tabular}

Values are presented as mean (standard deviation)

Multivariate ANCOVA was conducted with age and sex as covariates. ${ }^{\dagger} p<0.05,{ }^{*} p<0.01,{ }^{\dagger} p<0.001$.

PTSD, post traumatic stress disorder; MDD, major depressive disorder; SDNN, standard deviation of all RR intervals; RMSSD, square root of the mean squared differences of successive normal sinus intervals; VLF, very low frequency; LF, low frequency; HF, high frequency; TP, total power; ApEn, approximate entropy. 


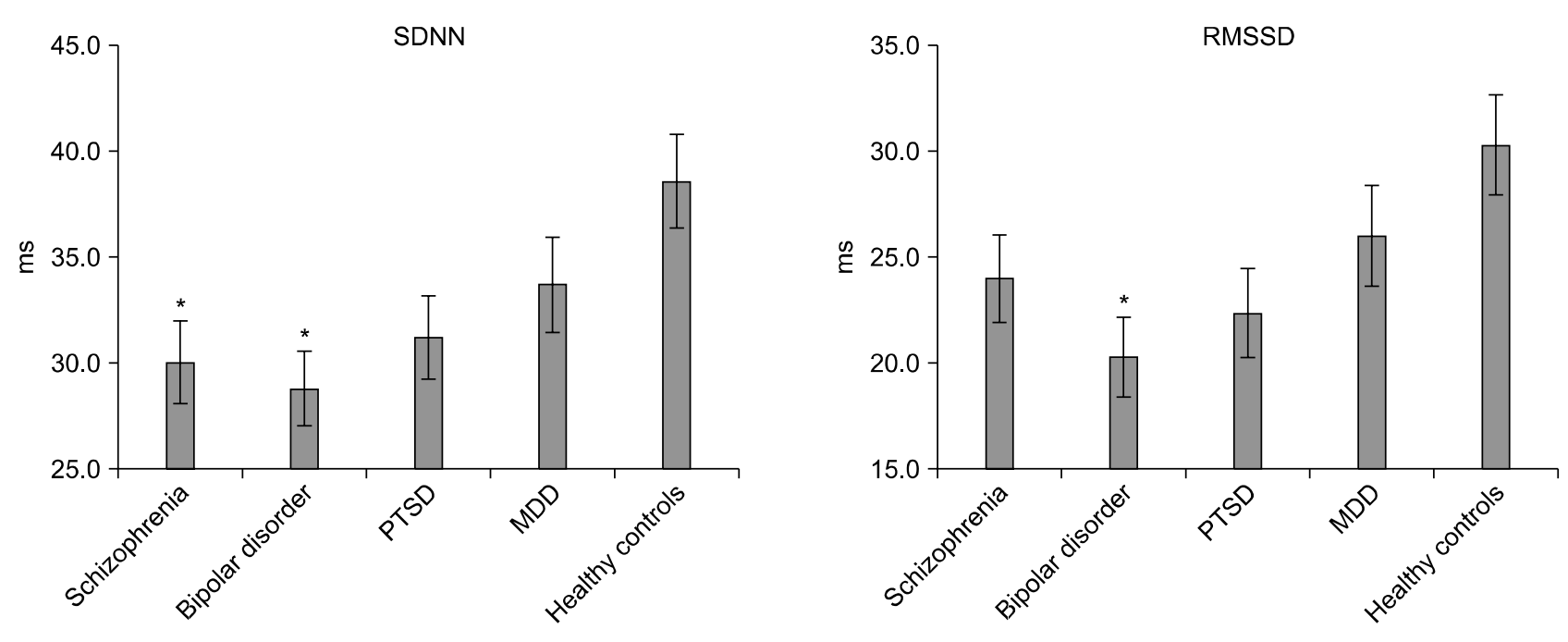

Fig. 1. Time domain analysis of heart rate variability (HRV) index. The standard deviation of all RR intervals (SDNN) of schizophrenia and bipolar disorder were significantly reduced compared to healthy controls. The square root of the mean squared differences of successive normal sinus intervals (RMSSD) of bipolar disorder was significantly reduced compared to healthy controls. *Significant differences compared to healthy controls $(p<0.05)$. PTSD, post-traumatic stress disorder; MDD, major depressive disorder.
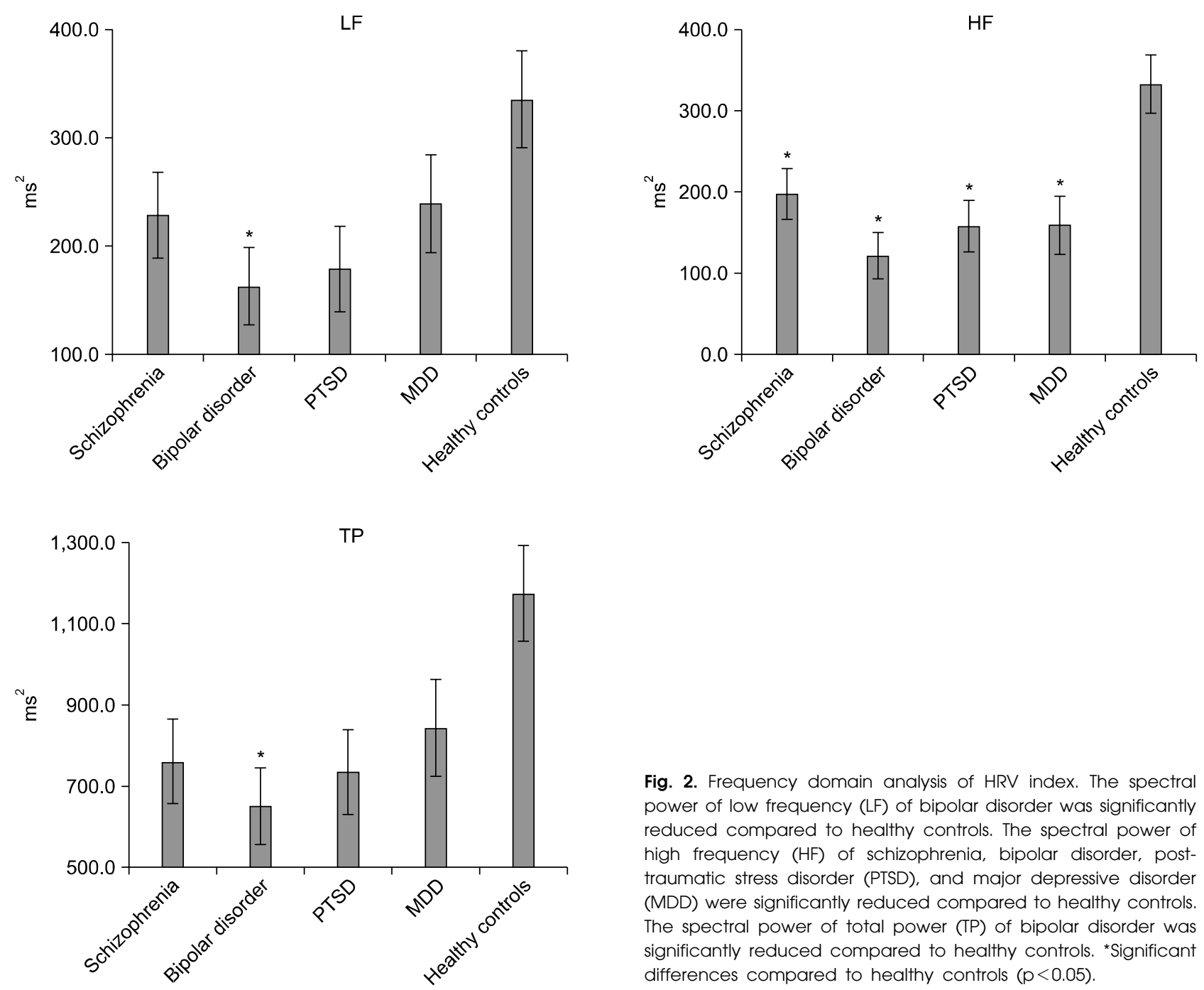

Fig. 2. Frequency domain analysis of HRV index. The spectral power of low frequency (LF) of bipolar disorder was significantly reduced compared to healthy controls. The spectral power of high frequency (HF) of schizophrenia, bipolar disorder, posttraumatic stress disorder (PTSD), and major depressive disorder (MDD) were significantly reduced compared to healthy controls. The spectral power of total power (TP) of bipolar disorder was significantly reduced compared to healthy controls. *Significant differences compared to healthy controls $(p<0.05)$. 
nificant differences in between-group comparisons: patients with schizophrenia versus healthy controls (estimated mean \pm standard error; $30.05 \pm 1.95$ vs. $38.57 \pm 2.22$, corrected $p=0.040)$ and patients with bipolar disorder versus healthy controls $(28.80 \pm 1.78$ vs. $38.57 \pm 2.22$, corrected $p=0.006$ ) (Fig. 1).

\section{RMSSD}

We found a significant main effect of RMSSD $(F[4$, $165]=3.17, p=0.015)$. Post hoc analysis revealed a significant difference in the between-group comparisons: patients with bipolar disorder versus healthy controls (20.30 \pm 1.89 vs. $30.27 \pm 2.35$, corrected $p=0.009$ ) (Fig. 1).

\section{VLF}

No significant main effect was observed.

\section{LF}

We observed a significant main effect of LF ( $F[4$, $165]=2.72, p=0.031)$. Post hoc analysis revealed a significant difference in between-group comparisons: patients with bipolar disorder versus healthy controls (163.04 \pm 35.99 vs. $335.48 \pm 44.70$, corrected $p=0.025)$ (Fig. 2).

\section{HF}

A significant main effect of HF was observed $(F[4$, $165]=5.86, p<0.000)$. Post hoc analysis revealed four significant differences in the between-group comparisons: patients with schizophrenia versus healthy controls (197.24 \pm 31.82 vs. $332.83 \pm 36.07$, corrected $p=0.048)$, patients with bipolar disorder versus healthy controls (121.35 \pm 29.04 vs. $332.83 \pm 36.07$, corrected $p<0.001$ ), patients with PTSD versus healthy controls (157.56 \pm 31.99 vs. $332.83 \pm 36.07$, corrected $p=0.004)$, and patients with MDD versus healthy controls $(159.16 \pm 36.27$ vs. $332.83 \pm 36.07$, corrected $p=0.014$ ) (Fig. 2).

\section{TP}

We found a significant main effect of TP $(F[4,165]=$ 3.38, $p=0.011$ ). Post hoc analysis revealed a significant difference in the between-group comparisons: patients with bipolar disorder versus healthy controls (651.42 \pm 95.33 vs. $1174.69 \pm 118.41$, corrected $p=0.006$ ) (Fig. 2).

\section{LH/HF}

No significant main effect was observed.

\section{ApEn}

No significant main effect was observed.

\section{DISCUSSION}

This study compared the HRV of patients with several psychiatric disorders (i.e., schizophrenia, bipolar disorder, PTSD, and MDD) with that of healthy controls. We found that SDNN and HF were significantly reduced in patients with schizophrenia compared with healthy controls. Patients with bipolar disorder showed the most decreased HRV function, and they also had significantly reduced SDNN, RMSSD, TP, LF, and HF compared with healthy controls. HF was significantly reduced in patients with PTSD compared with healthy controls.

HF was significantly reduced in patients with schizophrenia compared with healthy controls; however, no significant difference was observed in LF. The present results are consistent with previous studies. Bär et al. ${ }^{29)}$ and Valkonen-Korhonen et al. ${ }^{31)}$ revealed that HF was decreased in patients with schizophrenia patients compared with healthy controls, whereas LF was not. These data suggest that patients with schizophrenia have dysfunctional parasympathetic functioning and relatively preserved sympathetic functioning compared with healthy controls. RMSSD dysfunction also occurs in patients with schizophrenia. ${ }^{36)}$ RMSSD is supposedly related to parasympathetic activity, providing another measure of vagal tone. Therefore, decreased RMSSD in patients with schizophrenia suggests that this disorder is characterized by dysfunctional parasympathetic activity.

Patients with bipolar disorder demonstrated the most decreased HRV function, and they had significantly reduced SDNN, RMSSD, TP, LF, and HF compared with healthy controls. Previous studies are consistent with the present findings. Cohen et al. ${ }^{34)}$ examined HRV in euthymic bipolar subjects and reported a decrease in SDNN, a decrease in the $\mathrm{LF} / \mathrm{HF}$ ratio, and an increase in $\mathrm{HF}$ power compared with healthy subjects. Additionally, Migliorini et $a .^{37)}$ reported that patients with bipolar disorder had significantly decreased RMSSD and SDNN. According to Henry et al., ${ }^{35)}$ patients in the manic phase of bipolar disorder exhibit a significant decrease in HRV compared with age- and sex-matched healthy subjects. An increase in the ratio of sympathetic to parasympathetic activity is associated with more severe manic symptoms and unusual thought content, indicating that HRV alterations in bipolar disorder may be state-dependent. In our study, patients with bipolar disorder showed the most significant HRV 
dysregulation compared with patients with other kinds of psychiatric illnesses. Extreme affective lability, which is experienced by patients with bipolar disorder, may have contributed to the significant HRV dysregulation in these patients. From this perspective, the finding of decreased HRV during emotional regulation in subjects with high neuroticism reported by Di Simplicio et al. ${ }^{38)}$ supports our results. Further research on this issue is required.

HF was significantly reduced in patients with PTSD compared with healthy controls. The present results support previous studies. ${ }^{39)}$ According to Hauschildt et al. ${ }^{40)}$ the PTSD group had lower HRV than did non-trauma-exposed controls at baseline and throughout different affective conditions, implying decreased parasympathetic activity and inflexible response regulation. These results show that PTSD is related to the magnitude of the decrease in parasympathetic cardiac control during stress.

The patients with MDD in our study had no significant main change in HRV. However, in other studies, patients with MDD with and without comorbid anxiety had reduced HRV. Those with comorbid anxiety disorder showed the greatest reductions. ${ }^{41)}$ In another study, depression was also associated with reduced HRV, which decreases with the increasing severity of depression. ${ }^{10)}$

A recent study reported that vagal nerve stimulation (VNS) successfully improves depressive symptoms in treatment-resistant depression, ${ }^{42)}$ suggesting a strong relationship between sympathovagal dysregulation and depressive symptoms.

In sum, our results suggest that, among various psychiatric illnesses (i.e., schizophrenia, bipolar disorder, MDD, and PTSD), patient with bipolar disorder have the most decreased HRV function compared with healthy controls.

This study has several limitations. First, we did not exclude the medication effect. As antidepressants have different effects on HRV function, ${ }^{14,19-21)}$ medication effects should be carefully controlled in future studies. Second, the age effect could not be strictly controlled, and HRV is known to be influenced by age and sex differences. ${ }^{43,44}$ Indeed, elderly individuals tend towards a reduced HFHRV index compared with younger people. Moreover, men with depressive symptoms have reduced parasympathetic activity compared with control subjects, whereas no differences between depressed women and controls have been reported. ${ }^{44)}$ Because the demographic characteristics of patients with various psychiatric disorders (e.g., schizophrenia, bipolar disorder, PTSD, and MDD) differ, the recruitment of separate groups of healthy controls that are well matched to individuals with each psychiatric disorder is necessary to clarify the HRV data.

Despite these limitations, our study has value because we explored the HRV indices of those with various psychiatric disorders using the same study protocol. Our results do not support the use of HRV for discriminating among psychiatric illnesses. However, our results suggest that HRV, particularly HF-HRV, is a sensitive variable and can be used as a supplementary tool for discriminating between patients with psychiatric illnesses and healthy controls.

\section{Acknowledgments}

This research was supported by Basic Science Research Program through the National Research Foundation of Korea (NRF) funded by the Ministry of Education, Science and Technology (No. 2012R1A1A2043992).

\section{REFERENCES}

1. Haapaniemi TH, Pursiainen V, Korpelainen JT, Huikuri HV, Sotaniemi KA, Myllylä VV. Ambulatory ECG and analysis of heart rate variability in Parkinson's disease. J Neurol Neurosurg Psychiatry 2001;70:305-310.

2. Agelink MW, Majewski T, Wurthmann C, Lukas K, Ullrich $\mathrm{H}$, Linka $\mathrm{T}$, et al. Effects of newer atypical antipsychotics on autonomic neurocardiac function: a comparison between amisulpride, olanzapine, sertindole, and clozapine. J Clin Psychopharmacol 2001;21:8-13.

3. Berntson GG, Lozano DL, Chen YJ. Filter properties of root mean square successive difference (RMSSD) for heart rate. Psychophysiology 2005;42:246-252.

4. Pincus SM. Approximate entropy as a measure of system complexity. Proc Natl Acad Sci U S A 1991;88:2297-2301.

5. Yeragani VK, Pohl R, Mallavarapu M, Balon R. Approximate entropy of symptoms of mood: an effective technique to quantify regularity of mood. Bipolar Disord 2003;5:279286.

6. Kim JH, Yi SH, Yoo CS, Yang SA, Yoon SC, Lee KY, et al. Heart rate dynamics and their relationship to psychotic symptom severity in clozapine-treated schizophrenic subjects. Prog Neuropsychopharmacol Biol Psychiatry 2004;28:371-378.

7. Malliani A. Principles of cardiovascular neural regulation in health and disease. Kluwer Academic Pub;2000.

8. Levy MN. Autonomic interactions in cardiac control. Ann N Y Acad Sci 1990;601:209-221.

9. Ehrenthal JC, Herrmann-Lingen C, Fey M, Schauenburg H. Altered cardiovascular adaptability in depressed patients without heart disease. World J Biol Psychiatry 2010;11:586593.

10. Kemp AH, Quintana DS, Gray MA, Felmingham KL, Brown K, Gatt JM. Impact of depression and antidepressant treatment on heart rate variability: a review and metaanalysis. Biol Psychiatry 2010;67:1067-1074.

11. Rottenberg J. Cardiac vagal control in depression: a critical analysis. Biol Psychol 2007;74:200-211.

12. Tonhajzerova I, Ondrejka I, Javorka K, Turianikova Z, Farsky I, Javorka M. Cardiac autonomic regulation is im- 
paired in girls with major depression. Prog Neuropsychopharmacol Biol Psychiatry 2010;34:613-618.

13. Boettger S, Hoyer D, Falkenhahn K, Kaatz M, Yeragani VK, Bär KJ. Nonlinear broad band dynamics are less complex in major depression. Bipolar Disord 2008;10:276-284.

14. Koschke M, Boettger MK, Schulz S, Berger S, Terhaar J, Voss A, et al. Autonomy of autonomic dysfunction in major depression. Psychosom Med 2009;71:852-860.

15. Schulz S, Koschke M, Bär KJ, Voss A. The altered complexity of cardiovascular regulation in depressed patients. Physiol Meas 2010;31:303-321.

16. Voss A, Boettger MK, Schulz S, Gross K, Bär KJ. Genderdependent impact of major depression on autonomic cardiovascular modulation. Prog Neuropsychopharmacol Biol Psychiatry 2011;35:1131-1138.

17. Moser M, Lehofer M, Hoehn-Saric R, McLeod DR, Hildebrandt G, Steinbrenner B, et al. Increased heart rate in depressed subjects in spite of unchanged autonomic balance? J Affect Disord 1998;48:115-124.

18. Yeragani VK, Pohl R, Balon R, Ramesh C, Glitz D, Jung $\mathrm{I}$, et al. Heart rate variability in patients with major depression. Psychiatry Res 1991;37:35-46.

19. Udupa K, Thirthalli J, Sathyaprabha TN, Kishore KR, Raju TR, Gangadhar BN. Differential actions of antidepressant treatments on cardiac autonomic alterations in depression: A prospective comparison. Asian J Psychiatr 2011;4:100106.

20. Yeragani VK, Roose S, Mallavarapu M, Radhakrishna RK, Pesce V. Major depression with ischemic heart disease: effects of paroxetine and nortriptyline on measures of nonlinearity and chaos of heart rate. Neuropsychobiology 2002;46:125-135

21. Glassman AH, Bigger JT, Gaffney M, Van Zyl LT. Heart rate variability in acute coronary syndrome patients with major depression: influence of sertraline and mood improvement. Arch Gen Psychiatry 2007;64:1025-1031.

22. Mujica-Parodi LR, Korgaonkar M, Ravindranath B, Greenberg $\mathrm{T}$, Tomasi $\mathrm{D}$, Wagshul $\mathrm{M}$, et al. Limbic dysregulation is associated with lowered heart rate variability and increased trait anxiety in healthy adults. Hum Brain Mapp 2009;30:47-58.

23. Bleil ME, Gianaros PJ, Jennings JR, Flory JD, Manuck SB. Trait negative affect: toward an integrated model of understanding psychological risk for impairment in cardiac autonomic function. Psychosom Med 2008;70:328-337.

24. Pittig A, Arch JJ, Lam CW, Craske MG. Heart rate and heart rate variability in panic, social anxiety, obsessivecompulsive, and generalized anxiety disorders at baseline and in response to relaxation and hyperventilation. Int $J$ Psychophysiol 2013;87:19-27.

25. Wahbeh H, Oken BS. Peak high-frequency HRV and peak alpha frequency higher in PTSD. Appl Psychophysiol Biofeedback 2013;38:57-69.

26. Tan G, Dao TK, Farmer L, Sutherland RJ, Gevirtz R. Heart rate variability $(H R V)$ and posttraumatic stress disorder (PTSD): a pilot study. Appl Psychophysiol Biofeedback 2011;36:27-35.

27. Shaikh al arab A, Guédon-Moreau L, Ducrocq F, Molenda S, Duhem S, Salleron J, et al. Temporal analysis of heart rate variability as a predictor of post traumatic stress disorder in road traffic accidents survivors. $J$ Psychiatr Res 2012;46:790-796.

28. Cohen H, Kotler M, Matar MA, Kaplan Z, Miodownik H,
Cassuto Y. Power spectral analysis of heart rate variability in posttraumatic stress disorder patients. Biol Psychiatry 1997;41:627-629.

29. Bär KJ, Letzsch A, Jochum T, Wagner G, Greiner W, Sauer $\mathrm{H}$. Loss of efferent vagal activity in acute schizophrenia. $J$ Psychiatr Res 2005;39:519-527.

30. Boettger S, Hoyer D, Falkenhahn K, Kaatz M, Yeragani VK, Bär KJ. Altered diurnal autonomic variation and reduced vagal information flow in acute schizophrenia. Clin Neurophysiol 2006;117:2715-2722.

31. Valkonen-Korhonen M, Tarvainen MP, Ranta-Aho P, Karjalainen PA, Partanen J, Karhu J, et al. Heart rate variability in acute psychosis. Psychophysiology 2003;40: 716-726.

32. Okada T, Toichi M, Sakihama M. Influences of an anticholinergic antiparkinsonian drug, parkinsonism, and psychotic symptoms on cardiac autonomic function in schizophrenia. J Clin Psychopharmacol 2003;23:441-447.

33. Toichi M, Kubota Y, Murai T, Kamio Y, Sakihama M, Toriuchi $\mathrm{T}$, et al. The influence of psychotic states on the autonomic nervous system in schizophrenia. Int J Psychophysiol 1999;31:147-154.

34. Cohen H, Kaplan Z, Kotler M, Mittelman I, Osher Y, Bersudsky Y. Impaired heart rate variability in euthymic bipolar patients. Bipolar Disord 2003;5:138-143.

35. Henry BL, Minassian A, Paulus MP, Geyer MA, Perry W. Heart rate variability in bipolar mania and schizophrenia. $J$ Psychiatr Res 2010;44:168-176.

36. First M, Gibbon M, Spitzer R, Williams J, Benjamin LS. User's guide for the structured clinical interview for DSM-IV Axis II personality disorders. New York State Psychiatric Institute, Biometrics Research, New York 1997.

37. Kleiger RE, Bigger JT, Bosner MS, Chung MK, Cook JR, Rolnitzky LM, et al. Stability over time of variables measuring heart rate variability in normal subjects. Am J Cardiol 1991;68:626-630.

38. Migliorini M, Mendez MO, Bianchi AM. Study of heart rate variability in bipolar disorder: linear and non-linear parameters during sleep. Front Neuroeng 2011;4:22.

39. Di Simplicio M, Costoloni G, Western D, Hanson B, Taggart P, Harmer CJ. Decreased heart rate variability during emotion regulation in subjects at risk for psychopathology. Psychol Med 2012;42:1775-1783.

40. Hauschildt M, Peters MJ, Moritz S, Jelinek L. Heart rate variability in response to affective scenes in posttraumatic stress disorder. Biol Psychol 2011;88:215-222.

41. Kemp AH, Quintana DS, Felmingham KL, Matthews S, Jelinek HF. Depression, comorbid anxiety disorders, and heart rate variability in physically healthy, unmedicated patients: implications for cardiovascular risk. PLoS One 2012; 7:e30777.

42. George MS, Rush AJ, Marangell LB, Sackeim HA, Brannan $\mathrm{SK}$, Davis SM, et al. A one-year comparison of vagus nerve stimulation with treatment as usual for treatment-resistant depression. Biol Psychiatry 2005;58:364-373.

43. Abhishekh HA, Nisarga P, Kisan R, Meghana A, Chandran $\mathrm{S}$, Trichur Raju, et al. Influence of age and gender on autonomic regulation of heart. J Clin Monit Comput 2013; 27:259-264.

44. Garcia RG, Zarruk JG, Guzman JC, Barrera C, Pinzon A, Trillos E, et al. Sex differences in cardiac autonomic function of depressed young adults. Biol Psychol 2012;90: 179-185. 